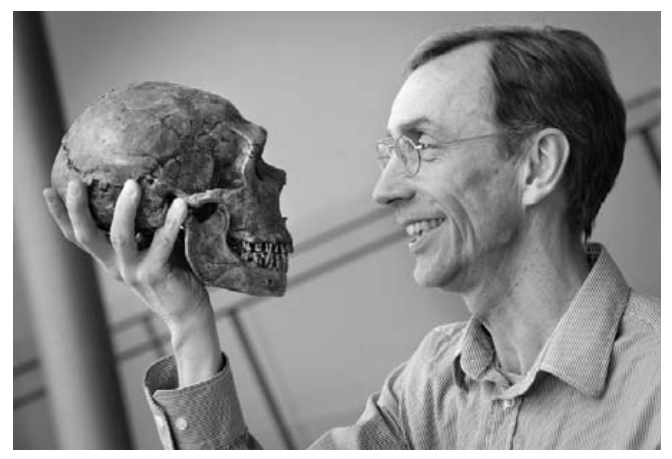

\title{
Dorcas Cummings Lecture
}

\author{
Svante PäÄвo
}

Dr. Svante Pääbo presented the Dorcas Cummings lecture entitled "The Genetic Legacy of Neanderthals" to friends and neighbors of Cold Spring Harbor Laboratory and Symposium participants on Saturday, May 30, 2015. Dr. Pääbo is director of the Department of Genetics at the Max Planck Institute for Evolutionary Anthropology. What follows is an abbreviated and edited transcript of his lecture.

Thank you for the honor of giving this lecture; it is a great pleasure for me to be here.

Our laboratory is interested in the Neanderthals. As you know, Neanderthals were a robust form of humans that appear in the fossil record of Europe and western Asia around 400 thousand years ago and existed in that part of the world until they disappeared about 30-40 thousand years ago, around the time modern humans appeared in Europe and Asia. There are at least two reasons why we should be interested in Neanderthals. The first is that they are the closest evolutionary relatives to all present-day humans. So if we want to define ourselves as fully modern humans, it's Neanderthals to whom we should compare ourselves. The other reason is that they disappeared just two or three thousand generations ago. So it's interesting to try to find out what happened to them and how they may have interacted with our direct ancestors.

Modern humans, with a skeleton indistinguishable from present-day people, first appeared in Africa. Around 100 thousand years ago, they spread to the Middle East and around 50-60 thousand years ago, they began spreading into Europe and the rest of Asia. It was then that they must have met Neanderthals in western Eurasia, and in East Asia other forms of now extinct humans that are less well known. A sometimes intense debate in paleontology for the last 30-40 years has centered on whether or not Neanderthals contributed genetically to modern humans. Some championed a "Total Replacement" model, where modern humans replaced Neanderthals without any mixing (i.e., $0 \%$ contribution). On the other extreme, others claimed that Neanderthals are the ancestors of present-day Europeans (i.e., a "Total Continuity" model). Between these two extreme hypotheses there is a continuum that encompasses in principle anything from very small to very large contributions.

We got a first chance to address this with the help of DNA studies in the early 1990 s, when we got access to a Neanderthal fossil, and not just any Neanderthal fossil but the Neanderthal fossil, the one that was found in a cave in the Neander Valley in 1856 and gave its name to this group of humans. That discovery was the first time people realized that other forms of humans had been around before present-day humans. When we obtained this unique bone sample we were fortunate to be able to draw on the 15 years of experience on working with ancient DNA. For example, we had collected experience on how to avoid or minimize contamination from presentday DNA from ourselves and from the chemicals or equipment we used.

We focused on the mitochondrial genome, a part of the genome that is present in many copies per cell and is therefore easier than other parts of the genome to retrieve. Using the techniques available at the time, we constructed a portion of the mitochondrial genome and it turned out to be very different from present-day humans. Whereas the mitochondrial genomes in present-day humans go back to a common ancestor somewhere 100-200 thousand years ago, you have to go back about half a million years to find an ancestor that shared a mitochondrial genome in common with Neanderthals. Since then, we-and othershave sequenced many mitochondrial genomes from $\mathrm{Ne}$ anderthals, and they all fall outside the variation of present-day humans so it is clear that there are no humans today that carry a Neanderthal mitochondrial genome. In terms of the mitochondrial genome, it's a total replacement. But the mitochondrial genome is a very tiny part of our total genome and is inherited only through the maternal lineage as a single unit. Therefore the full picture of our genetic relationships with Neanderthals can only be found in the nuclear genome.

I'm on the published record 10 years ago or so saying "We will never be able to sequence the nuclear genome of a Neanderthal; it's too degraded" or "There's too little DNA there; it can't be done." Of course, you should never say things like that, particularly in biology, because you will often be overtaken by technical developments. In this case, it was the development of high-throughput DNA sequencing at the beginning of the millennium that came along and allow millions of DNA molecules to be sequenced quickly and cheaply. It allowed us to extract 
the DNA from a fossil, sequence all of it, and compare it to the human genome.

The first time this approach worked for Neanderthals was on a bone from Vindija Cave in Croatia, in southern Europe. We used a small bone fragment, dated to about 38 thousand years ago. The first thing you notice when you look at the DNA in this bone is that it's in very short pieces, mostly in the 40-60-base-pair range. They trail off dramatically between 60 and 100 base pairs and there are virtually no fragments above 200 base pairs, whereas if you extract DNA from a blood sample from a person today, you can easily get pieces up to 10-20 thousand base pairs. The other noticeable issue is that very little of the total DNA we sequenced came from Neanderthals. Our very best bone samples only had on the order of $3 \%-4 \%$. All the rest were from fungi and bacteria that had colonized the bone over tens of thousands of years in the cave. We went on to extract DNA from many other samples - about 200 DNA extracts from 70 different fossils from 16 different excavation sites - to find the one that were best preserved. We focused ultimately on three bones from three different individuals found at the Vindija site and sequenced over a billion DNA fragments from those bones. Again, most of the sequences were bacterial and not from the Neanderthal, but the ones that were, we were able to match to the human genome that had by then been sequenced, taking into account typical errors seen in ancient DNA sequences.

By 2010, we had map sequences to a little over half of the Neanderthal genome and we could begin to ask questions. One of our first questions was "What happened when modern humans met Neanderthals? Was there interbreeding?" If Neanderthals contributed to the ancestors of present-day Europeans, then Europeans today should share more genetic variants with Neanderthals than Africans do. Because Neanderthals were never in Africa, Africans could not have picked up any genetic contribution from them. In other words, if the total replacement model is correct, a Neanderthal should be equally distinct from people in Africa as they are from modern Europeans. If on the other hand there was mixing, then on average a Neanderthal will share more genetic variants with Europeans. To resolve this, we sequenced a number of present-day human genomes: one European (we chose a French person as our "typical" European), two Africans (a Yoruba from West Africa and a San from Southern Africa), a Han person from China, and one person from Papua New Guinea. Then we did a very simple test. We looked for all the positions in pairs of the present-day humans where their genomes differed, then checked these positions against the Neanderthal sequence to see how often there were matches to each of the two present-day individuals. As we expected, the two Africans did not differ significantly from each other in their degree of matching to the Neanderthal. When we then did the same comparison between the French person and the Yoruba against the Neanderthal, to my surprise, we found statistically greater matching to the European individual. Even more surprising to me was that when we compared a Chinese individual and an African individu- al, we again saw more matching to the Chinese person, even though Neanderthals were probably never in China. Similarly, in the genome from Papua New Guinea, where we are sure Neanderthals had never been, we again saw more matching between Neanderthals and that individual than between Neanderthals and Africans. The model that arose from these results suggested that modern humans that left Africa mixed with Neanderthals, perhaps in the Middle East where we know there were Neanderthals, and then went on to became the ancestors of everyone outside Africa. If that happened, their descendants would have carried with them a Neanderthal genetic component out into the rest of the world, to the extent that somewhere between 1 and 2 percent of your genome, if your roots are outside Africa, come from Neanderthals.

The next important question was to determine when this mixing occurred. There are genetic ways of estimating that but those are model-dependent, and I'm a fan of direct evidence. Two years ago, we finally got a chance to directly begin to look at when admixture happened when a person looking for mammoth tusks along the Irtysh River in the Ust'-ishim district of western Siberia found a human bone. It looked like a modern human humerus and when we radiocarbon-dated it, we were surprised to find that it was about 45 thousand years old. This is actually the oldest directly dated modern human bone outside the Middle East and Africa that we know of. When the person from whom this bone came lived, Neanderthals existed so we could ask if the ancestors of this individual had already mixed with Neanderthals or not. The answer was yes, but not only that. The fragments of DNA that matched the Neanderthal sequence tended to be longer than today. This is precisely what you would you expect. If you imagine a Neanderthal and a modern human having a baby, that baby would of course have one chromosome from the Neanderthal parent and one from the modern human parent. If this individual then continued to mate with modern humans, there would be recombination in every generation such that the Neanderthal contribution would be broken up and become smaller and smaller in each successive generation. The smaller the pieces are, the longer ago they came into the gene pool. You can then look at correlations of Neanderthal variants along the chromosomes of modern humans and the 45-thousandyear-old individual and estimate that Neanderthal DNA entered the gene pool a little over 300 generations before this individual lived. That's somewhere on the order of 50-60 thousand years ago, which fits well with when we believe modern humans left Africa.

One of the limitations of our research back in 2010 was that we had a very incomplete Neanderthal genome. Over the last 2 years, we've worked with Russian colleagues, Anatoly Derevyanko and his team, who excavate the Denisova cave site in the Altai Mountains of southern Siberia near the border with Mongolia. They found a small toe bone in 2010 that turned out to come from a Neanderthal. It was extremely well preserved, and we had developed our methodology to the point that we could produce a very high-quality genome from this specimen. Instead of having fragments that covered just a little over 
half the genome, we could now cover all of the approximately two-thirds of the genome to which it is possible to map the short DNA fragments that survived in the bone. Since we sequence these regions many times over we can even discern the two variants at the positions where this Neanderthal individuals inherited different genetic variants from her mother and her father.

With such a complete genome sequence, we can go through the present-day human genomes and identify which pieces are likely to come from Neanderthals. It confirms our previous conclusion that somewhere between 1 and 2 percent of the genome of any modern non-African individual is derived from Neanderthal ancestors, although which Neanderthal DNA pieces a person carries varies considerably from individual to individual. You might ask if we should think about this as a lot of Neanderthal DNA that we carry, or little. One way to get perspective on this is to remind yourself that you share $50 \%$ of your DNA with each of your parents, $\sim 25 \%$ with a grandparent and so on. If you go back six generations, on average you'll have $1.5 \%$ of your DNA from a particular ancestor in that generation. So the one or two percent Neanderthal DNA many of us carry is, if you like, as if you had one Neanderthal ancestor six generations back in your family tree. But it's distributed differently across the genome than if it came from just one direct ancestor six generations back. Instead of a few large stretches of DNA making up about $1 / 64$ th of your genome, the Neanderthal DNA contribution is distributed in small fragments scattered throughout the genome. Another question is how much of the total Neanderthal genome persists in people living today. If we look across multiple sequences from modern humans, we can piece together longer pieces of the Neanderthal genome. The jury is still out how much in total can be puzzled together, but at least on the order of $40 \%$, and probably a bit more, of the Neanderthal genome is still to be found in people today.

But Denisova Cave in Siberia has offered even more fascinating insights. In 2008, the Derevianko team found a tiny bone, even smaller than that Neanderthal bone. It was the tip of the last phalanx of the pinky of a child. We were able to eventually determine a high-quality sequence from that fragment but already when we had a rough draft of it we were quite surprised to find that it did not come from a Neanderthal. It was something else, something that shared an ancestor with Neanderthals far back in time, probably about 400,000 years. We called this new group of extinct humans Denisovans after the Denisova Cave where they were first found. Just as with Neanderthals, we can ask if they contributed to modern humans. Surprisingly, we find that there are quite significant Denisovan contributions to modern human DNA, but not in Siberia but in the Pacific. On the order of $5 \%$ of the genomes of people in Papua New Guinea or the Aborigines of Australia come from Denisovans. Obviously, we don't think the ancestors of people in Australia were in Siberia, but rather that the Denisovans were more widespread in the past, such that the ancestors of the Pacific populations met them somewhere in Southeast Asia.
Let's summarize what we think we now know from genome studies about the origin of Neanderthals and Denisovans and modern humans. A common ancestor for Neanderthals and Denisovans lived somewhere in Africa about half a million years ago. They spead out of Africa and then evolved in western Eurasia to what we call Neanderthals and in eastern Eurasia to what we call Denisovans. At that time, there were other forms of humans there too. We know that Homo floresiensis, the "hobbits," were in Indonesia, for example, and probably other human forms too. Modern humans arose in Africa later and started spreading out of Africa and the Near East around 50-60 thousand years ago, mixing with Neanderthals probably somewhere in the Middle East before continuing throughout Eurasia. There's now suggestive evidence that the ancestors of present-day people in East Asia mixed with Neanderthals again, adding a little bit of Neanderthal DNA to their genomes. Some also mixed with Denisovans in Southeast Asia and continued on to the Pacific. The Neanderthals and Denisovans then became extinct, but live on a little bit in the form of $1 \%-2 \%$ of the genomes of modern humans of non-African ancestry, and up to $7 \%$ percent of the genomes of people in Melanesia. In the future, we might find other groups that also contributed, in China, for example, and also in Africa where modern humans of course spread across the continent. There are some indications from present-day genetic variation that this may be the case. So in summary, while we can rule out the Total Replacement model for modern human origins, it is nevertheless the case that the big picture is one of replacement. Neanderthals, Denisovans, and other human forms that modern humans encountered disappeared; so to keep this perspective we like to use the term "Leaky Replacement" for how we now think the spread of modern humans across Eurasia occurred.

Let's now turn our attention to what the genetic contribution from Neanderthals to modern humans may means functionally. If one looks across genomes from present-day humans one finds pieces of Neanderthal DNA that occur at a high frequency among people today. Some fragments are present in 70\%-80\% of contemporary individuals and some are shared among Asians and Europeans, while some are unique to Asia and some unique to Europe. These genetic variants may have been positively selected in the past and one group of genes that stand out among these regions encodes keratins, structural proteins of skin and hair. We don't know why this is so, but in the future we'll likely find that some aspect of how the hair and skin looks or functions in Europeans and Asians come from Neanderthals.

More importantly, Peter Parham's group at Stanford showed that genes that encode transplantation antigens, which are important for how our immune system responds to different antigens, come from Neanderthals and Denisovans. A group in China discovered that genes involved in lipid catabolism often come from Neanderthals in Europe, whereas this is not the case in Asia. A gene encoding a transmembrane lipid transport protein, $S L C 16 A 11$, has been found to carry two variants that 
differ drastically from each other in the modern population. One variant, which is frequent in Asians and in Native Americans (up to 25\%), comes from Neanderthals and confers a risk for Type 2, or adult-onset, diabetes. It may seem surprising that something that contributes to disease comes from Neanderthals and rises to high frequency. This is likely an example of the common phenomenon that genetic variants have different functional consequences depending on the environment in which they find themselves. The variant that causes diabetes today could have been advantageous when the availability of food was limited in the past. The SLC16A11 variant might therefore well have been a Neanderthal adaptation to starvation that today has negative consequences when many people have ample nutrition throughout their lives.

Denisovans have also made functionally important contributions to the genomes of contemporary humans. At high altitude in Tibet, many people carry a genetic variant of the EPAS1 gene that regulates how many red blood cells are present in the blood and how they transport oxygen. Last year, Rasmus Nielsen's group from Berkeley showed that this variant of the EPAS1 gene, which is very rare elsewhere, has been contributed by Denisovans to the ancestors of Tibetans.

There is a picture emerging that suggests that when modern humans emerged from Africa, they met other forms of humans such as Neanderthals and Denisovans, who had by then lived for hundreds of thousands of years in the environments of Eurasia. These other forms of humans had adapted to those environments and carried genetic variants that were advantageous. When modern newcomers from Africa then mixed with these resident populations they sometimes pick up advantageous genetic variants that then rose in frequency. Such a scheme is called call "adaptive introgression" and is described in species other than humans.

Another interesting question to ask is what genetic variants did not come over from Neanderthals. In other words: What of the Neanderthal genetic legacy did modern humans reject from their gene pool? We can look across the genomes of present-day people for regions where we statistically would expect Neanderthals to have contributed, but yet we find little or no contributions. We can then ask, for example, where in the body the genes located in those regions are expressed. The tissue that then stands out are the testicles, the male germline. That could hint at some problem with regard to male fertility in $\mathrm{Ne}$ anderthal-modern human hybrids. It is common in other closely related groups of species that mate that the heterogametic sex, the males, have problems with fertility. In mules, for example, the offspring of donkeys and horses, the females are often fertile but the males not. It could thus be that modern humans and Neanderthals were on the verge of becoming reproductively isolated when they met.
Another area of interest are the genetic variants that are unique to modern humans relative to Neanderthals and Denisovans - that is, the variants that changed after we diverged from the Neanderthal lineage and then came to be present in all or almost modern humans. Such variants may be very interesting because they may help us understand how modern humans are different from Neanderthals and other earlier forms of humans. Neanderthals lived for 400 thousand years or so. Their stone tools, at least to my eyes, look very similar at the beginning of their history and at the end of their history. Modern humans have existed a little over 100 thousand years and you don't need to be an archaeologist to see that the technology today is very different from 100 thousand years ago. So modern humans differed from other human forms that lived before them in that they created technology that changes rapidly. Figurative art, art that depicts recognizable objects, only appears with modern humans, and of course, modern humans spread across open water; they came to the Americas, to Australia, to every little island in the Pacific_-something earlier forms of humans did not.

With the help of the Neanderthal genome we can now catalog those genetic differences that are found in most or all humans today but not present in Neanderthals and Denisovans. Interestingly, this is not a very long catalog; only a little over 30 thousand single-nucleotide changes and small insertions and deletions. Unfortunately, we are still very bad at saying what any particular change means functionally. Nevertheless, the challenge for the next decade is to find out which of these changes may have been crucial to create the biological foundation that made modern human culture and human history possible. This work has just begun. It involves the testing of genetic variants unique to modern humans in animal models and in genetically engineered stem cells as well as the study of rare mutations in people today.

I thank you for your attention.

\section{ACKNOWLEDGMENTS}

Very many people have been involved in this work, more than I can mention. I would like to give special recognition to a few who are currently crucial to our work. Matthias Meyer developed the ultrasensitive method of making DNA libraries that made it possible to produce high-coverage Neanderthal and Denisovan genomes. Kay Prüfer coordinated the effort to analyze the high-coverage Neanderthal genome, and Janet Kelso organizes all the bioinformatics much of the research in our department. Monty Slatkin and his group at Berkeley and David Reich and his group in Boston are crucial for much of the population genetic analyses. 


\section{$\$_{\text {CSH }}^{\infty}$ Cold Spring Harbor Symposia SYMPOSIA On Quantitative Biology}

\section{Dorcas Cummings Lecture}

Svante Pääbo

Cold Spring Harb Symp Quant Biol 2015 80: 291-294

Access the most recent version at doi:10.1101/sqb.2015.80.030171

\section{License}

Email Alerting Receive free email alerts when new articles cite this article - sign up in Service the box at the top right corner of the article or click here. 